\title{
MINING SPATIOTEMPORAL PATTERNS OF THE ELDER'S DAILY MOVEMENT
}

\author{
C. R. Chen ${ }^{a *}$, C. F. Chen ${ }^{a}$, M.E. Liu ${ }^{\text {b }}$, S.J. Tsai ${ }^{b, c}$, N. T. Son ${ }^{\text {a }}$, L.V. Kinh ${ }^{\text {a }}$ \\ ${ }^{a}$ Center for Space and Remote Sensing Research, National Central University, Taoyuan, Taiwan 32001 ccruncu@gmail.com, \\ cfchen@csrsr.ncu.edu.tw, ntsonait@ hotmail.com, khinlau@yahoo.com \\ b Department of Psychiatry, Taipei Veteran General Hospital, Taipei, Taiwan 11217 liumuen@ gmail.com, tsai610913@gmail.com \\ ${ }^{\mathrm{c}}$ Psychiatric Division, School of Medicine, National Yang-Ming University, Taipei, Taiwan 11221
}

Commission II, WG II/8

KEY WORDS: Geographic Information System (GIS), wearable device, daily movement pattern

\begin{abstract}
:
With rapid developments in wearable device technology, a vast amount of spatiotemporal data, such as people's movement and physical activities, are generated. Information derived from the data reveals important knowledge that can contribute a long-term care and psychological assessment of the elders' living condition especially in long-term care institutions. This study aims to develop a method to investigate the spatial-temporal movement patterns of the elders with their outdoor trajectory information. To achieve the goal, GPS based location data of the elderly subjects from long-term care institutions are collected and analysed with geographic information system (GIS). A GIS statistical model is developed to mine the elderly subjects' spatiotemporal patterns with the location data and represent their daily movement pattern at particular time. The proposed method first finds the meaningful trajectory and extracts the frequent patterns from the time-stamp location data. Then, a density-based clustering method is used to identify the major moving range and the gather/stay hotspot in both spatial and temporal dimensions. The preliminary results indicate that the major moving area of the elderly people encompasses their dorm and has a short moving distance who often stay in the same site. Subjects' outdoor appearance are corresponded to their life routine. The results can be useful for understanding elders' social network construction, risky area identification and medical care monitoring.
\end{abstract}

\section{INTRODUCTION}

\subsection{General Instructions}

Aging population has become a global issue. In Taiwan, according to official survey data, the older population is $12 \%$ in 2015. Furthermore, it will increase drastically to $20 \%$ in 2020 (National Development Council, 2014). To develop a good health care monitoring system that can cope with the blooming aging population becomes one of the critical polices. With the rapid development of technology and science, wearable device is now popular for application. High performances, such as rich information collection, low electrical consumption, and high computation capability, etc., become affordable and easy to unitize for many applications. Mobility is important as older people being able to remain independent. Studies find that reduced mobility can restrict a person's social life as well as limiting their access to shops, leisure and other activities. Daily moving patterns obtained from daily routines can be very useful for helping elderly people who suffer from any kind of cognition decline (Hsiao et al., 2011; Kamihira et al., 2013). Satellite-based GPS technology offers potential advantages for studying movement in the community/residential area, which may involve outdoor walking, as well as the historical dailytrajectory. GPS receivers, which are capable of providing information of how often elderly people leave their homes, when they leave their homes, where they travel, how they travel (on foot or in vehicle), and how quickly they move. It offers promise for ultimately being able to objectively monitor community mobility in older adults (Krenn et al., 2011). They can also collect detailed spatiotemporal information about individual trips away from home that cannot be obtained with existing questionnaires (Webber and Porter, 2009).
This study aims to investigate the spatial-temporal patterns of the elderly people's daily movement from their outdoor trajectory information. The GPS-based wearable devices are delivered to the subjects to record their daily movements. A GIS model will be developed to mine their daily moving patterns.

\section{METHODOLOGY}

\subsection{Study site}

This study is designed to carry out in a government-owned senior citizens' residential home near the suburban area of New Taipei city, Taiwan. The total area of the study site is about 4.7 ha. There are four main dormitory areas in which depended on their need of health-care condition. The residents can walk around the community at any time. Those who need less health care and have good mobility are arranged to live in Dormitory area I.

\subsection{Subject selection}

This study focuses on the spatiotemporal moving behaviour of older people. Thus, subjects with good mobility (resided in area I) and aged above 70 years old are selected in this study. Subjects has worn the trackers for 4 months (December 2015 March 2016). They have been visited every 2 days for tracker diagnose and battery changing, and interviewed for the psychological assessment every 2 weeks.

\subsection{Tracking human movements with GPS-based tracker}

GPS tracker is designed as a small and thin size which can be stored in the pockets or hanged on belts for the subject's 
convenience. The equipped GPS chip has the ability of concurrent reception of GPS/QZSS, GLONASS, BeiDou which has a wide GNSS signal coverage and a faster position acquisition. Sensed data is transmitted by $3 \mathrm{G}$ network and the time interval of each sensed data transition is at least 20 seconds. All the data is collected and stored by Geographical Information System (GIS) server. For the developing purpose, research team can use XMPP protocol to have bidirectional communication for each tracker. The protocol is used to provide tracker's current status, such like GPS NEMA information, battery time and G-sensor status that indicates the moving events triggered by the wearer' mobility.
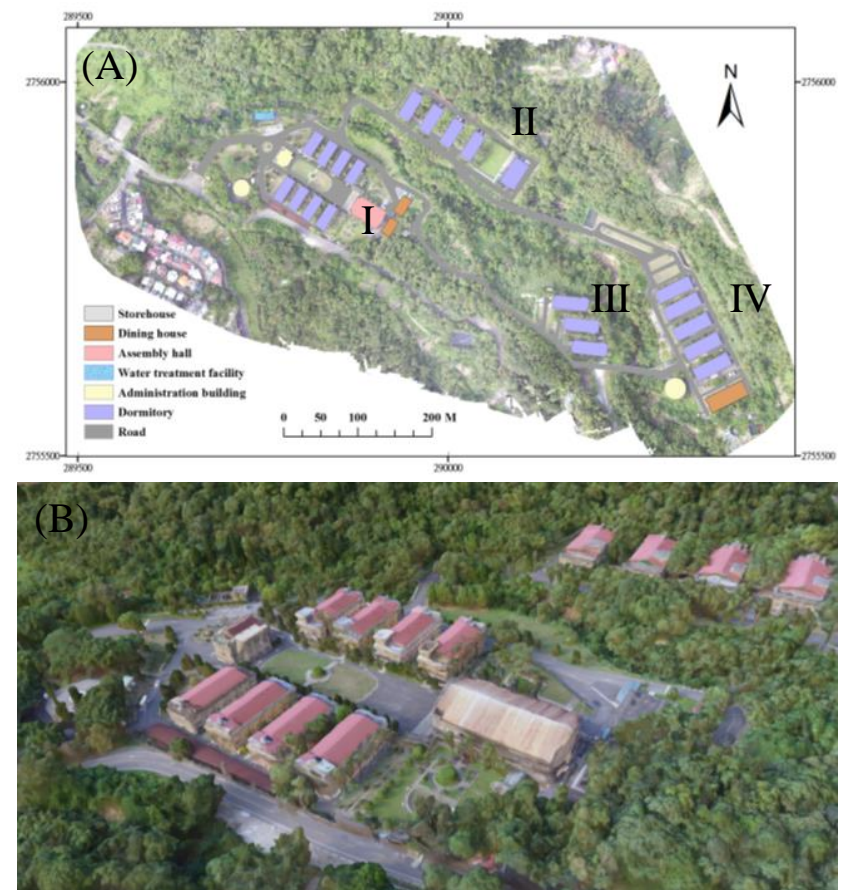

Figure 1. (A)The overview map of the study site (map projection: TWD 97); (B) The $2.5 \mathrm{D}$ map of the dormitory area I.

\subsection{Sensor Data Processing}

Sensed data received by GIS server is the location information of each subject. A location can be represented in absolute, relative, and symbolic (home, administrator office, nursing facility) form. Meanwhile, a location can refer to three geospatial interpretations: 1) A point location, 2) a region, and 3 ) a trajectory. In order to reduce the error of GPS data, the differential GPS method with fixed stations are implemented, and the stored point information are analysed in raster formation. $10 \mathrm{~m}$ spatial resolution are selected in this study. The minimum monitoring window is set to 1 hour for the periodic mobility analysis within a day.

\subsection{Quantifying human movement parameters.}

In order to mine spatiotemporal patterns of the elder's daily movements, the variables, such as location, speed, duration time longer-stay sites, gather site (the area where the subjects gather at the same time), and implicitly social connection (who, when, where and how long) are processed and stored in the GIS database. An assumption is made for the implicitly social connection that two subjects gather together within a predefined distance threshold $(20 \mathrm{~m}$ or within the neighboring cell) at the same time with at least 10 minutes stay.

\subsection{Mining daily movement patterns of the elders}

Processed data will be extracted from different time window. The longer-term GPS data are used with the Fuzzy clustering and sequential pattern to identify trends, and regular occurrences of the daily movement. The procedure includes (1) to construct the trajectory information (2) to extract the frequent patterns from the time-stamp location (3) to use density-based clustering method to identify their major moving range and the hotspot with their geographical location in both spatial and temporal dimensions. The $20 \%$ number of subjects with the higher total daily outdoor hour (so-called High group), and the $20 \%$ number of subjects with the lower total daily outdoor hour (so-called Low group) are selected for the comparison. A weekly in-situ observation is proceed to identify the mining results.

\section{RESULTS AND DISCUSSION}

\subsection{Daily movement events}

The hourly average of daily movement for all subjects is shown in Figure 2. According to Figure 2, there are three peak time periods during outdoor movement. It may be the matching of the restaurant food service hour (6:30 AM, 11 AM and 4:30 PM). Both High group and Low group have similar peak patterns in a day. High group have more outdoor hours than the low-group, and they may stay longer outside in the morning.

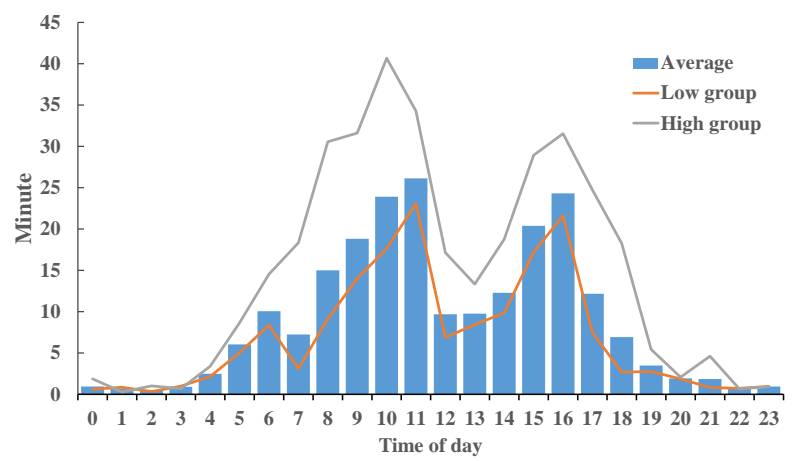

Figure 2. The outdoor time of daily movement per hour. The "High" represents the $20 \%$ of the highest total daily outdoor hour, while the "Low" represents $20 \%$ of lowest total daily outdoor hour from all subjects' data.

It was found that the spatial range of the mobility of elderly people is severely restricted in most home-absent time spending in close proximity to their residences. To explain the spatial patterns for subjects, the subject ID01 with highest daily outdoor time and ID04 with lowest daily outdoor time are selected to demonstrate. Figure 3 shows ID01 and ID04 (from the same dormitory). Their movements are quantified by the percentage of their time -spending in one day. The dinning path which subjects go to the dinning house at specific time under a necessary time are excluded in this study. According to the analysed results, although ID01 has the most outdoor hours with a larger appearance area, the major moving area compared to ID04 which has the least daily outdoor hours has similar spatial pattern: the area are usually closer to their dormitory; and they usually stay at one point outside. Also, it can be observed that the outdoor hours of subjects do not correspond to their moving distance. Identified by field observation when the research team go for interviews, some of the subjects only 
sit outside for a long time. Some stand steadily to smoke then go directly back indoors.

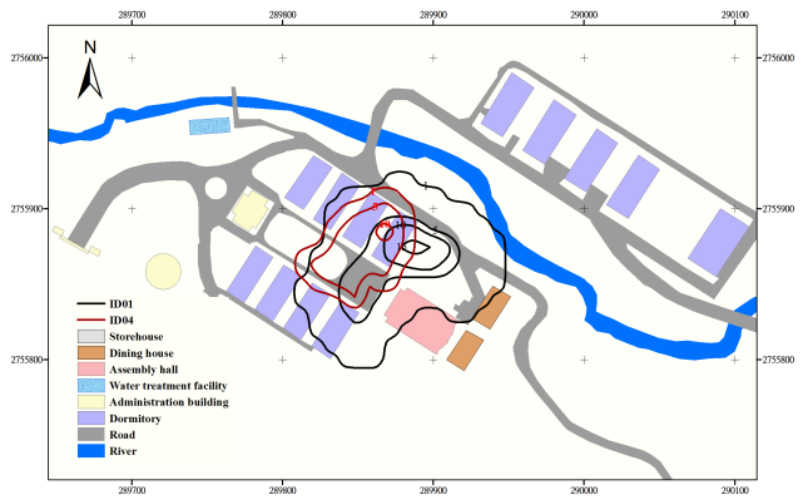

Figure 2. The temporal location map of Subjects' daily movements. The contour value represents the percentage of staying time in a day for one subject.

Another interesting phenomenon is also observed, that the subjects have the least total outdoor appearances are often alone when they are outside. However, without enough subjects' information and experimental test, the results will acquire more supporting evidence and data to be convinced .

\section{CONCLUSION}

The high-resolution spatial and temporal data enables detailed analysis of the differences in timing and distance of the participants' daily outdoor mobility patterns. The mobility of elderly people with a GPS elderly tracking device can be observed, verifying their staying in a particular area such as home and other locations. All information can be accessed anytime since elderly tracking systems can function and operate on a continuous basis. The accumulated data is useful to create a basic-line pattern. Another beneficial feature that elderly GPS tracking information contains is the ability to set virtual boundaries and to detect the irregular moving path. The preliminary finding will be integrated with subjects' psychological assessment data and weather information in the future. The results will be useful for understanding elders' social network construction, risky area identification and medical care monitoring.

\section{ACKNOWLEDGEMENTS}

Data support is provided by Department of Psychiatry, Taipei Veteran General Hospital. Mr. Yo-Cheng Kuo is acknowledged for UAS photo processing and Ms Wen-Pai Chih for field data collections.

\section{REFERENCES}

Hsiao, C.-C., Sung, Y.-J., Lau, S.-Y., Chen, C.-H., Hsiao, F.-H., Chu, H.-H., Huang, P., 2011. Towards long-term mobility tracking in NTU hospital's elder care center, Pervasive Computing and Communications Workshops (PERCOM Workshops), 2011 IEEE International Conference on. IEEE, pp. 649-654.

Kamihira, I., Nakajima, T., Matsumura, T., Miura, H., Ono, T., 2013. Abnormality Detection of Persons Living Alone Using
Daily Life Patterns Obtained from Sensors. World Academy of Science, Engineering and Technology, International Journal of Computer, Electrical, Automation, Control and Information Engineering 7, 1545-1549.

Krenn, P.J., Titze, S., Oja, P., Jones, A., Ogilvie, D., 2011. Use of global positioning systems to study physical activity and the environment: a systematic review. American Journal of Preventive Medicine 41, 508-515.

National Development Council, 2014. Population Projections for R.O.C. (Taiwan): 2014 2060.

Webber, S.C., Porter, M.M., 2009. Monitoring mobility in older adults using global positioning system (GPS) watches and accelerometers: a feasibility study. J Aging Phys Act 17, 455467. 